\title{
Small dense and desialylated low density lipoprotein in diabetic patients
}

\author{
Igor A. Sobenin ${ }^{1,2}$, Elena V. Galitsyna ${ }^{3,4}$, Andrey V. Grechko ${ }^{5}$, Alexander N. Orekhov ${ }^{2,4}$ \\ ${ }^{1}$ Department of Cardiovascular Pathology, Russian Cardiology Research and Production Complex, 121552 Moscow, Russia. \\ ${ }^{2}$ Laboratory of Angiopathology, Institute of General Pathology and Pathophysiology, 125315 Moscow, Russia. \\ ${ }^{3}$ Department of Genetics, Southern Federal University, 344090 Rostov-on-Don, Russia. \\ ${ }^{4}$ Institute for Atherosclerosis Research, Skolkovo Innovative Center, 121609 Moscow, Russia. \\ ${ }^{5}$ Federal Scientific Clinical Center for Resuscitation and Rehabilitation, 109240 Moscow, Russia.
}

Correspondence to: Dr. Igor A. Sobenin, Russian Cardiology Research and Production Complex, 15-a 3-rd Cherepkovskaya Str, 121552 Moscow, Russia. E-mail: igor.sobenin@gmail.com

How to cite this article: Sobenin IA, Galitsyna EV, Grechko AV, Orekhov AN. Small dense and desialylated low density lipoprotein in diabetic patients. Vessel Plus 2017;1:29-37.

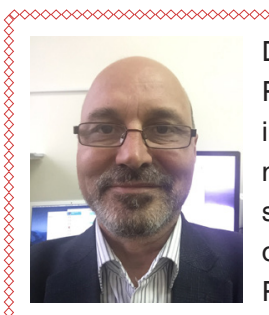

Dr. Igor A. Sobenin is a Leading Researcher at the Department of Cardiovascular Pathology at Russian Cardiology Research and Production Complex, Moscow, Russia. He has received his MD in Internal Diseases in 1988, PhD in Endocrinology in 1991, and DSc in Pathophysiology and Biochemistry in 2006. His research activity is related to molecular, biochemical and cellular mechanisms of atherosclerosis, including genetic and phenotypic markers of susceptibility, clinical, epidemiological and population studies in the field of chronic diseases with a special emphasis on atherosclerosis. He has published more than 140 papers in international peer-reviewed journals indexed by PubMed, Scopus and Web of Science.

Article history:

Received: 26-10-2016

Accepted: 29-12-2016

Published: 31-03-2017

Key words:

Diabetes mellitus,

atherosclerosis,

low density lipoprotein,

desialylated LDL,

small dense LDL,

electronegative LDL

\begin{abstract}
Aim: This study was undertaken to investigate the physicochemical properties of modified low density lipoprotein (LDL) in diabetes. Methods: LDL from 10 type 1 and 10 type 2 diabetic patients, as well as LDL from 10 non-diabetic subjects, was subdivided into bound and nonbound fractions by affinity chromatography on Ricinus communis agglutinin-agarose, and further characterized by sialic acid content, hydrated density, electrophoretic mobility, and the ability to induce cholesterol deposition in cultured cells. Results: The non-bound LDL fraction was similar to native LDL from healthy subjects, with respect to its physicochemical properties, and did not produce intracellular cholesterol accumulation. The bound LDL fraction was characterized by several alterations differentiating it from non-bound LDL, namely, significantly lowered sialic acid content (by 35-50\%, compared with non-bound LDL), increased electrophoretic mobility (by 40-50\%), increased hydrated density (difference in modae, 5.6-5.9 mg/mL), and smaller particle size (difference in modae, 3.8-4.9 nm). Bound LDL possessed the ability to induce a 2.1 - to 2.7 -fold increase in intracellular cholesterol content. Conclusion: The results showed the presence of a dense, small, more electronegative, desialylated LDL subfraction in the blood of diabetic patients, which is in vivo modified atherogenic LDL.
\end{abstract}

This is an open access article licensed under the terms of Creative Commons Attribution 4.0 International License (https://creativecommons.org/licenses/by/4.0/), which permits unrestricted use, distribution, (c) (i) in any medium, as long as the original author is credited and the new creations are licensed under the 


\section{INTRODUCTION}

Accelerated coronary and peripheral vascular atherosclerosis is the most common long-term complication of diabetes mellitus. ${ }^{[1-3]}$ The mortality rate of coronary heart disease (CHD) is up to four times higher in diabetic than nondiabetic individuals. Therefore, CHD is the leading cause of death in diabetic patients. ${ }^{[4-6]}$ Many factors contribute to the increased rate of atherosclerosis progression in diabetes, including alterations in plasma lipid profile, platelet function, clotting factors, metabolism of arterial wall cells, and elevated blood pressure. The precise mechanisms of premature atherogenesis in diabetic patients, however, remain unclear.

At the cellular level, the deposition of intracellular cholesterol in the arterial wall and subsequent foamcell formation is a typical feature of early atherosclerotic lesions. ${ }^{[7]}$ Low-density lipoprotein (LDL) has been associated with sourcing of accumulating lipids. ${ }^{[6,8,9]}$ However, LDL isolated from healthy individuals failed to produce notable cholesterol accumulation in cultured arterial smooth muscle cells or macrophages. ${ }^{[10,11]}$ It was hence accepted that LDL is required to undergo structural alterations or chemical modifications to become atherogenic. ${ }^{[12]}$ However, modified LDL particles are still not considered as clinical biomarkers or therapeutic targets because of insufficient evidence. Therefore, additional studies, both basic and clinical, are necessary to fill the existing gap in the knowledge.

Previously, we have shown that LDL from diabetic patients, unlike LDL from healthy individuals, is able to induce significant lipid deposition in cultured cells derived from uninvolved (non-atherosclerotic) human aortic intima. ${ }^{[13]}$ LDL from diabetic patients was subdivided into two fractions by lectin chromatography on Ricinus communis agarose, wherein bound (desialylated) LDL showed substantial dissimilarity with non-bound (native) LDL with respect to chemical composition and atherogenic properties, i.e. it was just a fraction of in vivo modified LDL presumably responsible for lipid accumulation in cultured cells. ${ }^{[13,14]}$

In the past few years, it was demonstrated that even in healthy individuals, LDL is heterogeneous in size and hydrated density, and the presence of small dense LDL (sdLDL) in blood is associated with a higher risk of clinical manifestations of atherosclerosis. ${ }^{[15-17]}$ We have shown that multiple-modified atherogenic LDL occurring in blood of atherosclerotic patients is characterized also by increased hydrated density, and therefore may be easily regarded as sdLDL. ${ }^{[18]}$

This study was undertaken to obtain more information on the desialylated LDL isolated from the blood of diabetic patients and healthy individuals, to examine their distribution by hydrated density and the relationship between the LDL density and atherogenicity.

\section{METHODS}

\section{Patients}

This study was conducted in accordance with the Helsinki Declaration of 1975 as revised in 1983. It was approved by the local ethics committee of the Institute for Atherosclerosis Research, Skolkovo Innovation Center, Moscow, Russia. All participants gave their written informed consent prior to inclusion in the study. The study group comprised of 10 type 1 diabetic patients, 10 type 2 diabetic patients, and 10 healthy control subjects, free from coronary artery disease [Table 1]. The diagnosis of diabetes mellitus was verified according to the 1997 criteria by the Expert Committee on the Diagnosis and Classification of Diabetes Mellitus (ADA), 1998 WHO consultation criteria, and 1985 WHO criteria. ${ }^{[19]}$ Type 1 diabetic patients were on insulin therapy, and type 2 diabetic patients were treated with oral hypoglycemic agents, namely sulfonylurea derivatives.

LDL isolation, lectin chromatography, and density fractionation

Venous blood (15 mL) was drawn after overnight fasting in plastic tubes containing $0.1 \%$ EDTA. Plasma was separated by centrifugation, and $\varepsilon$-aminocaproic acid $(1 \mathrm{mmol} / \mathrm{L}$ ) was added. LDL (density, 1.025$1.063 \mathrm{~g} / \mathrm{mL}$ ) was isolated by sequential preparative ultracentrifugation according to Lindgren ${ }^{[20]}$ in a Beckman L8-55 ultracentrifuge (Beckman Instruments Inc., Palo Alto, CA) using Type 50Ti fixed angle rotor operated at $40,000 \mathrm{~g}$ at $10^{\circ} \mathrm{C}$, and sterilized by filtration (pore size, $0.45 \mu \mathrm{m}$ ). The LDL preparations were dialyzed against 2,000 volumes of phosphate buffered saline (PBS) at $\mathrm{pH} 7.4$, overnight at $4{ }^{\circ} \mathrm{C}$. LDL was subfractionated into two fractions [non-bound (sialylated) LDL and bound

Table 1: Demographic findings and subject characteristics of study groups

\begin{tabular}{|c|c|c|c|}
\hline Characteristic & $\begin{array}{l}\text { Healthy } \\
\text { subjects }\end{array}$ & $\begin{array}{c}\text { Type } 1 \text { diabetic } \\
\text { patients }\end{array}$ & $\begin{array}{l}\text { Type } 2 \text { diabetic } \\
\text { patients }\end{array}$ \\
\hline Gender, M/F & $4: 6$ & $4: 6$ & $5: 5$ \\
\hline Age, years & $32.3(2.1)$ & $46.0(17.7)$ & $56.3(3.5)^{\star}$ \\
\hline Diabetes, years & - & $24.7(17.0)$ & $16.0(6.1)$ \\
\hline Glycemia, mmol/L & $4.6(0.3)$ & $9.1(2.1)^{*}$ & $11.3(1.8)^{\star}$ \\
\hline $\mathrm{TG}, \mathrm{mmol} / \mathrm{L}$ & $1.6(0.2)$ & $1.7(0.6)$ & $2.2(0.4)$ \\
\hline Cho, $\mathrm{mmol} / \mathrm{L}$ & $4.7(0.2)$ & $5.2(1.0)$ & $5.5(0.8)$ \\
\hline HDL-Cho, mmol/L & $1.3(0.1)$ & $1.4(0.2)$ & $1.3(0.3)$ \\
\hline
\end{tabular}

*Significant difference from healthy subjects, $P<0.05$. TG: plasma triglycerides; Cho: plasma cholesterol; HDL-Cho: plasma highdensity lipoprotein-cholesterol 
(desialylated) LDL] using affinity chromatography on Ricinus communis agglutinin ( $\mathrm{RCA}_{120}$ )-agarose (Sigma Chemical Co., St. Louis, MO) as described elsewhere. ${ }^{[21]}$ Briefly, approximately $2 \mathrm{mg}$ of LDL (by protein) was applied on the column containing $2 \mathrm{~mL}$ of the affinity gel equilibrated with PBS. Non-bound (sialylated) LDL was washed from the gel with 30 volumes of PBS containing $0.5 \mathrm{~mol} / \mathrm{L} \mathrm{NaCl}$, and bound (desialylated) LDL was eluted with 5 volumes of PBS containing $50 \mathrm{mmol} / \mathrm{L}$ galactose (Sigma Chemical Co., St. Louis, MO). LDL fractions were adjusted with solid $\mathrm{NaBr}$ to a density of $1.065 \mathrm{~g} / \mathrm{mL}$ and concentrated by ultracentrifugation. For cell culture experiments, aliquots of LDL preparations were dialyzed against PBS and sterilized by filtration (pore size, $0.45 \mu \mathrm{m}$ ). The larger portions of LDL fractions were subdivided in a density gradient as described elsewhere. ${ }^{[22,23]}$ Briefly, total, non-bound, and bound LDL were dialyzed against 2000 volumes of $\mathrm{NaCl} /$ $\mathrm{NaBr}$ solution with a density of $1.050 \mathrm{~g} / \mathrm{mL}$ overnight at $4{ }^{\circ} \mathrm{C}$. The gradients were formed into $14 \times 95-\mathrm{mm}$ tubes by successively layering $2.6 \mathrm{~mL}$ of $\mathrm{NaCl} / \mathrm{KBr}$ solutions having densities of 1.1289, 1.0637, 1.0500 (containing the LDL sample), 1.0398 , and $1.0282 \mathrm{~g} / \mathrm{mL}$. The gradients were centrifuged for $42 \mathrm{~h}$ in a Beckman L-80 ultracentrifuge (Beckman Instruments Inc., Palo Alto, CA) using Type SW40Ti swinging bucket rotor operated at $36,000 \mathrm{~g}$ at $10^{\circ} \mathrm{C}$. After centrifugation, the gradients were fractionated by upward displacement with $\mathrm{KBr}$ solution (density, $1.300 \mathrm{~g} / \mathrm{mL}$ ) at a speed of $0.5 \mathrm{~mL} / \mathrm{min}$ (Isco Density Gradient Fractionator, Model 640 , Isco, Lincoln, NE). The absorbance of the effluent was monitored with an Isco UA-5 absorbance detector (Isco, Lincoln, NE) at $280 \mathrm{~nm}$, and $0.4 \mathrm{~mL}$ fractions were collected. Equal salt gradients without LDL were run and fractionated simultaneously, and the densities of these fractions were measured to reconstruct the density profile of the gradient (DMA 45 Calculating Digital Density Meter, Anton Paar, Graz, Austria).

\section{Cell culture studies}

Subendothelial cells were isolated from uninvolved human aortic intima by dispersion with $0.15 \%$ collagenase..$^{[7]}$ Subendothelial cells represented a mix of typical smooth muscle cells (about $30-40 \%$ ), atypical (pericyte-like or stellate) smooth-muscle cells (about $40-45 \%$ ), resident macrophages (3-8\%), and very small number of cells that migrated from circulation (monocytes and lymphocytes). Because atherosclerosis develops only in the subendothelial intima, this type of primary culture is adequate for reproducing in vitro the major traits of atherogenesis at the cellular level (e.g. lipid deposition, proliferation, fibrosis, and inflammation), although this model has certain limitations with the respect to inter-experiment standardization. These limitations were avoided when the whole set of experiments was performed in the primary culture obtained from one autopsy sample. The results were reproduced twice in primary cultures obtained from two other autopsy samples and, hence, considered reliable. The autopsy material was taken from men aged 52-62 years who had died suddenly in an accident. The cells were suspended in Medium 199 containing standard additives: $2 \mathrm{mmol} / \mathrm{L}$ L-glutamine, $100 \mathrm{U} / \mathrm{mL}$ penicillin, $100 \mu \mathrm{g} / \mathrm{mL}$ streptomycin, $2.5 \mu \mathrm{g} / \mathrm{mL}$ Fungizone, and $10 \%$ fetal calf serum (FCS). Cells were seeded onto 24-well tissue culture plates at a density of $3 \times 10^{4}$ cells $/ \mathrm{cm}^{2}$ of growth area, and cultured at $37{ }^{\circ} \mathrm{C}$ in a humidified $\mathrm{CO}_{2}$-incubator $(95 \%$ air and $5 \%$ $\mathrm{CO}_{2}$ ). The medium was changed every other day. On the 7 th day in culture, the total, non-bound, and bound LDL fractions $(50 \mu \mathrm{g} / \mathrm{mL})$ were added in quadruplicate in Medium 199 containing 10\% human lipoproteindeficient serum (LDS). Control cells were incubated in Medium 199 containing 10\% LDS without LDL addition. After $24 \mathrm{~h}$ of incubation, cells were rinsed thoroughly with PBS, and cellular lipids were extracted thrice with n-hexane/isopropanol (3:2, v/v) according to Hara and Radin's protocol. ${ }^{[24]}$ Total cholesterol in the extracts was determined enzymatically. ${ }^{[25]}$ Cellular protein was measured according to Lowry et al. ${ }^{[26]}$

\section{Electrophoresis}

The aliquots of LDL samples ( $20 \mu \mathrm{g}$ LDL by protein) were run on agarose gel films (Ciba Corning Diagnostics $\mathrm{GmbH}$, Giessen, Germany) according to Lipid Research Clinics Manual of Laboratory Operations. ${ }^{[27]}$ The same LDL sample from healthy donors was used as the reference sample throughout all experiments, and its mobility was assumed as 1.00 . Nondenaturing polyacrylamide gradient gel electrophoresis was run on continuous $2-16 \%$ gradient gels as described elsewhere. ${ }^{[28]}$ After gel staining with Coomassie Brilliant Blue R-250, LDL-migration distance was measured by scanning densitometry at $560 \mathrm{~nm}$. LDL particle size was calculated according to the calibration curve reconstructed from migration distances of standards with known diameters using Exponential Curve Fit utility in the Sigmaplot 12.0 program package.

\section{Other analyses}

LDL sialic acid content was measured according to Warren's modified method. ${ }^{[29]}$ LDL protein was measured according to Lowry et al. ${ }^{[26]}$

\section{Statistical methods}

Results are reported as mean and standard deviation (SD). Significance of differences was evaluated using ANOVA and Mann-Whitney tests (IBM SPSS 20.0 statistical program package), and $P$ values $<0.05$ were considered statistically significant. 


\section{RESULTS}

\section{Sialic acid content of LDL fractions}

Sialic acid content of total LDL preparations accounted for 40.0 (SD 3.0) nmol/mg LDL protein in non-diabetic individuals, 32.9 (SD 2.8) nmol/mg in type 1, and 26.4 (SD 3.9) $\mathrm{nmol} / \mathrm{mg}$ in type 2 diabetic patients. Sialic acid content in diabetic patients' LDL was significantly lower than non-diabetic individuals $(P=0.027)$. Upon LDL sub-fractionation into non-bound and bound fractions by affinity chromatography on $\mathrm{RCA}_{120}$-agarose, nonbound LDL in all studied groups were characterized by high levels of sialic acid, quite comparable to normal values [41.5 (SD 1.7), 38.7 (SD 1.4), and 35.1 (SD 1.8) $\mathrm{nmol} / \mathrm{mg}$ LDL protein for non-diabetic individuals, type 1 , and type 2 diabetic patients, respectively; $(P>0.1)$. However, bound LDL had significantly lower sialic acid levels than total LDL $(P=0.029)$ and non-bound LDL $(P$ $=0.009$ ) in all studied groups; the levels accounted for 32.3 (SD 2.5), 24.8 (SD 4.0), and 17.9 (SD 2.3) nmol/mg LDL protein for non-diabetic individuals, type 1 , and type 2 diabetic patients, respectively. Thus, the obtained LDL fractions (non-bound and bound) differed significantly in sialic acid content and might be readily regarded as sialylated and desialylated LDL.

\section{Density of sialylated and desialylated LDL}

The density distributions of sialylated and desialylated LDL are shown in Figure 1. In non-diabetic individuals, the density peak of desialylated LDL was slightly shifted to the higher density region compared with that of sialylated LDL [1.0350 (SD 0.0003) vs. 1.0335 (SD $0.0003) \mathrm{g} / \mathrm{mL}, P=0.013$ ]. A more prominent shift was observed for desialylated LDL from type 1 diabetic patients and furthermore for desialylated LDL from type 2 diabetic patients [1.0395 (SD 0.0006 ) vs. 1.0338 (SD 0.0004), $P<0.001$, and 1.0408 (SD 0.0004) vs. 1.0350 (SD 0.006) g/mL, $P<0.001$, respectively]. Therefore, desialylated LDL was significantly denser than sialylated LDL, and this difference in LDL density was most evident in diabetic patients. It is also notable that even sialylated LDL in diabetic patients seemed to be slightly more dense than that in non-diabetic individuals [Figure 1], but this difference was not statistically significant.

Atherogenic potential of sialylated and desialylated LDL: correlation with LDL density

LDL atherogenicity was determined as the ability to induce cholesterol deposition in cells cultured from unaffected human aortic intima. As shown in Table 2, LDL from non-diabetic individuals did not affect cellular cholesterol content; neither did sialylated LDL, but desialylated LDL fraction increased intracellular cholesterol level moderately and significantly $(P$
$=0.037)$. The same difference was observed for sialylated and desialylated LDL from type 1 and type 2 diabetic patients; however, in some cases, sialylated LDL seemed slightly atherogenic, and desialylated LDL caused extensive cholesterol accumulation, much more than that of desialylated LDL from non-diabetic individuals [Table 2]. The effect of total LDL from diabetic patients appeared to have intermediate value between that of sialylated and desialylated LDL [Table 2],
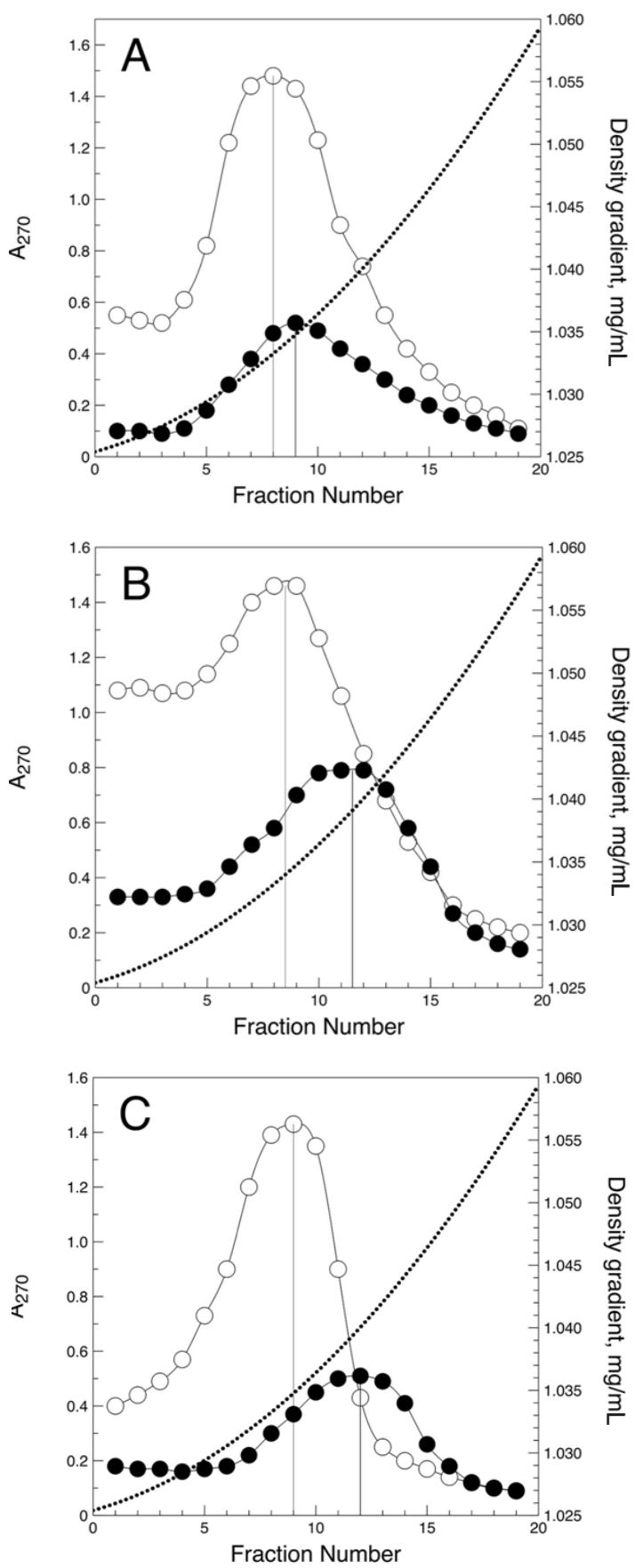

Figure 1: Density distribution of sialylated (hollow circles) and desialylated (filled circles) low-density lipoprotein in healthy subjects (A), type $1(B)$, and type 2 diabetic patients (C). Y-axis denotes optical density at $\lambda=270 \mathrm{~nm}$. Dotted line denotes the density gradient 
and was supposedly dependent on the proportion of desialylated (atherogenic) LDL in total preparation and of the extent of its desialylation.

The effects of sialylated and desialylated LDL fractionated by density on intracellular cholesterol content was also studied. The results from the experiment performed using LDL subfractions obtained from type 1 and type 2 diabetic patients are shown in Figure 2. The LDL samples from all patients produced similar but quantitatively different effects. Sialylated LDL did not induce an increase in the intracellular cholesterol content after $24 \mathrm{~h}$ of incubation under the cell culture conditions. However, the densest sialylated LDL subfractions $(\mathrm{d}>1.042 \mathrm{~g} / \mathrm{mL})$ produced a moderate but significant rise in intracellular cholesterol level. Subfractions of less dense desialylated LDL (1.025$1.035 \mathrm{~g} / \mathrm{mL}$ ) also produced no significant atherogenic effect, but LDL of greater density stimulated 1.3- to 2.3-fold cholesterol accumulation $(P<0.05)$. Denser
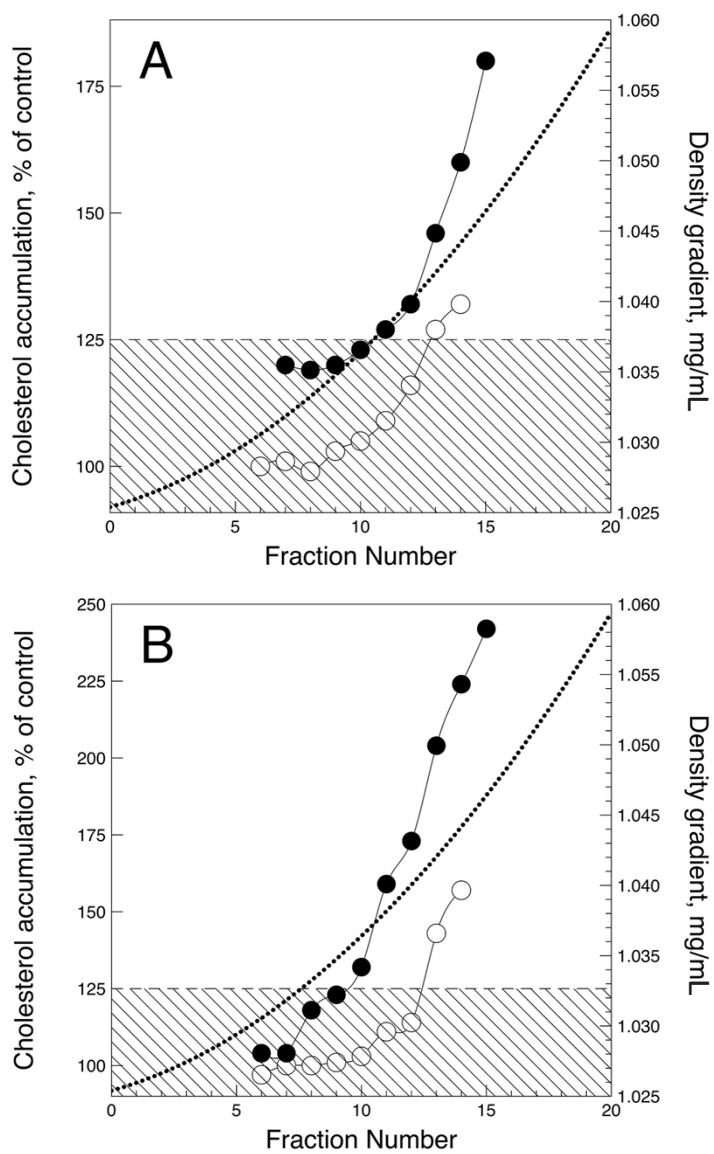

Figure 2: Atherogenic effects of sialylated (hollow circles) and desialylated (filled circles) LDL density subfractions from type $1(A)$ and type 2 (B) diabetic patients. Atherogenic effect was assessed as the ability of $\operatorname{LDL}(50 \mu \mathrm{g} / \mathrm{mL})$ to induce significant increase in the cholesterol content of subendothelial cells cultured from normal human aortic intima. Intracellular cholesterol content is expressed as the percentage of cholesterol level in control cells. The rectangular shaded areas denote the range of statistically insignificant changes in intracellular cholesterol level. LDL: low-density lipoprotein desialylated LDL subfractions from type 2 diabetic patients were much more effective than that from type 1 diabetic patients, with respect to intracellular cholesterol accumulation.

\section{Size of sialylated and desialylated LDL}

The size of total, sialylated, and desialylated LDL particles was assessed according to their migration distance in polyacrylamide gradient gel electrophoresis performed under non-denaturing conditions. As shown in Table 3, in every studied group, desialylated LDL particles appeared significantly smaller than the those in the total LDL preparation and more so than those in the sialylated LDL fraction. However, there was no significant difference in LDL size between non-diabetic individuals, type 1, and type 2 diabetic patients, with respect to either total LDL, sialylated LDL, or desialylated LDL.

\section{Electrophoretic mobility of sialylated and desialylated LDL}

The surface net charge of sialylated and desialylated LDL was judged according to their electrophoretic mobility in agarose gel. As shown in Table 4, desialylated LDL fractions in both non-diabetic and diabetic patients had higher mobility than sialylated LDL fractions, and this difference was statistically significant among diabetic patients $(P=0.041)$. Sialylated LDL from diabetic patients also seemed to have higher mobility than sialylated LDL from normal controls, but this difference was not statistically significant [Table 4].

Table 2: The effect of total, sialylated and desialylated LDL on intracellular cholesterol level

\begin{tabular}{|c|c|c|c|}
\hline \multirow{2}{*}{ Group } & \multicolumn{3}{|c|}{ Cholesterol accumulation, $\%$ above control } \\
\hline & Total LDL & Sialylated LDL & Desialylated LDL \\
\hline Healthy subjects & $5(3)$ & $3(3)$ & $26(9)^{\star \dagger \ddagger}$ \\
\hline $\begin{array}{l}\text { Type } 1 \text { diabetic } \\
\text { patients }\end{array}$ & $47(10)^{*}$ & $9(2)^{\dagger}$ & $110(45)^{\star \dagger \ddagger}$ \\
\hline $\begin{array}{l}\text { Type } 2 \text { diabetic } \\
\text { patients }\end{array}$ & $100(29)^{*}$ & $19(10)^{\dagger}$ & $169(33)^{\star \ddagger \ddagger}$ \\
\hline
\end{tabular}

*Significant intracellular cholesterol accumulation, $P<0.05$ vs control; ${ }^{\dagger}$ significant difference from total LDL, $P<0.05$; ${ }^{\ddagger}$ significant difference from sialylated LDL, $P<0.05$. LDL: low-density lipoprotein

Table 3: The size of total, sialylated and desialylated LDL particles

\begin{tabular}{lccc}
\hline \multirow{2}{*}{ Group } & \multicolumn{3}{c}{ LDL particle size, $\mathbf{n m}$} \\
\cline { 2 - 4 } & \multicolumn{2}{c}{ Total LDL } & Sialylated LDL Desialylated LDL \\
\hline Healthy subjects & $25.4(1.2)$ & $26.6(1.4)$ & $23.1(0.9)^{\star^{\dagger}}$ \\
$\begin{array}{l}\text { Type 1 diabetic } \\
\text { patients }\end{array}$ & $26.6(1.9)$ & $27.2(1.0)$ & $22.5(0.6)^{\star^{\dagger}}$ \\
$\begin{array}{l}\text { Type 2 diabetic } \\
\text { patients }\end{array}$ & $25.1(0.7)$ & $26.3(1.2)$ & $22.3(0.5)^{\star^{\dagger}}$ \\
\hline
\end{tabular}

*Significant difference from total $\mathrm{LDL}, P<0.05$; ${ }^{\dagger}$ significant difference from sialylated LDL, $P<0.05$. LDL: low-density lipoprotein 
Table 4: Relative electrophoretic mobility of sialylated and desialylated LDL fractions

\begin{tabular}{lcc}
\hline \multirow{2}{*}{ Group } & \multicolumn{2}{c}{ Relative LDL mobility } \\
\cline { 2 - 3 } & Sialylated LDL & Desialylated LDL \\
\hline Healthy subjects & $1.00(0.07)$ & $1.27(0.19)$ \\
Type 1 diabetic patients & $1.05(0.10)$ & $1.55(0.17)^{\star}$ \\
Type 2 diabetic patients & $1.29(0.21)$ & $1.85(0.29)^{*}$ \\
\hline
\end{tabular}

*Significant difference from sialylated LDL, $P<0.05$. LDL: lowdensity lipoprotein

\section{DISCUSSION}

We have shown previously that blood sera taken from diabetic patients, in contrast to sera from nondiabetic individuals, can induce cholesterol deposition in cultured cells derived from uninvolved human aortic intima, mainly because of LDL. ${ }^{[13]}$ LDL particles carry a variety of modifications, which are usually inherently atherogenic, unlike native LDL. ${ }^{[10]}$ The increase of a fraction of modified LDL can cause disruption of lipid metabolism and contribute to the development of atherosclerotic and metabolic diseases such as diabetes. According to Tsai et al., ${ }^{[30]}$ modified LDL levels were significantly higher in stroke survivors, thus attributing it as a risk factor for stroke outcome.

LDL particles in humans are heterogeneous in a polysaccharide moiety, and contain a single molecule of apolipoprotein B-100 per particle, and also 80-100 molecules of secondary proteins, approximately 1,500 molecules of esterified and non-esterified cholesterol, and a varying amount of other lipids. ${ }^{\left[{ }^{31]}\right.}$ It is possible to divide the LDL pool into two different sub-fractions [sialylated (sialic acid-rich) LDL and desialylated (sialic acid-poor) LDL]. Such separation is possible by using a column with $\mathrm{RCA}_{120}$ immobilized on $\mathrm{CNBr}$ activated agarose. ${ }^{[12,21]}$ Compared with sialylated LDL, desialylated LDL particles are smaller in size and contain more oxysterols, and less phospholipids and antioxidants. ${ }^{[12]}$ In terms of the ability to induce intracellular deposition of lipids, the desialylated LDL fraction is atherogenic. ${ }^{[12,23,32]}$ Several studies have reported an elevation of sialic acid serum levels in CHD patients, and also on its correlation with the severity of the coronary lesions. ${ }^{[33]}$ Diabetic patients' LDL, by the first approach, appeared to have a decreased level of sialic acid, and further investigations have shown that this was due to an increased proportion of desialylated LDL fraction in patients' blood and a greater extent of its desialylation. ${ }^{[13,14]}$ This LDL fraction was also characterized by increased non-enzymatic glycation and altered lipid composition, namely, decreased content of esterified cholesterol and elevated level of lyso-phospholipids. ${ }^{[13,14]}$ Taken together, these findings indicate that this LDL fraction with multiple modifications has marked atherogenic potency. In the present study, desialylated LDL from diabetic patients was additionally examined by density, particle size, and electrophoretic mobility to obtain a more complete physicochemical characterization of this atherogenic LDL fraction.

The density distribution profile of desialylated LDL from diabetic patients differed from that of sialylated LDL, evidently owing to the presence of denser particles. While this difference in LDL density between sialylated and desialylated LDL was also observed in nondiabetic individuals, it was markedly distinct among diabetics. Desialylated LDL was also characterized by diminished particle size, approximately by 1.2 -fold as compared with sialylated LDL.

It has been shown that sdLDL was characterized as highly atherogenic, and its level strongly correlated with cardiovascular disease risk. ${ }^{[15,16]}$ The presence of sdLDL is claimed to be highly associated with atherosclerosis development, and several studies have attempted to identify the risk of ischemic atherosclerotic events according to LDL subclass pattern. ${ }^{[15,16,18]}$ The higher prevalence of small dense LDL has been associated with cardiovascular events, acute ischemic stroke onset, and short-term mortality after acute ischemic stroke. ${ }^{[33-36]}$ Our data demonstrate that in the blood of the same patient, there are at least two distinct forms of LDL differing by particle size and density. Obviously, the LDL pattern would strongly depend on what LDL fraction prevails in circulation.

The most prominent feature of desialylated LDL is its ability to induce intracellular cholesterol accumulation. ${ }^{[12,13,23,32]}$ We have found previously that LDL atherogenicity in CAD patients and diabetic patients correlates negatively with sialic acid levels. ${ }^{[13]}$ Indeed, in vitro studies have shown that desialylated LDL treated with neuraminidase, which removes the sialic acid residues, results in a significant increase in LDL potency to induce lipid accumulation in cultured monocyte-macrophages or intimal cells. ${ }^{[32]}$ However, whether the loss of sialic acid in circulating LDL is the primary reason for their atherogenicity, remains unclear. The so-called desialylated LDL is characterized by several alterations to its chemical composition and physical properties, low sialic acid level being only one attribute of a wider scope of changes including the density, size, and electric charge of particles. ${ }^{[12,18,23]}$

Desialylated LDL showed higher mobility on agarose gel electrophoresis, thereby indicating that this LDL fraction has an elevated surface net charge, i.e. is a more electronegative LDL particle. ${ }^{[18]}$ It is definitely known that numerous techniques of in vitro LDL modification (e.g. 
acetylation, metal-dependent oxidation, methylation, malonic dialdehyde treatment, etc.) lead to the formation of anionic LDL thus rendering it atherogenic. ${ }^{[31]}$ It is possible that LDL surface charge plays a significant role in the processes of lipoprotein-to-cell interaction, and charge alterations may substantially modify LDL cellular metabolism, thus resulting in lipid deposition. The elevated electrophoretic mobility of desialylated LDL may be readily explained considering the significantly reduced content of free amino groups of desialylated apoB. ${ }^{[30,31]}$

A smaller size and increased density of desialylated LDL particles seem to be typical attributes of modified atherogenic LDL. ${ }^{18,31]}$ In our study, LDL's ability to induce intracellular cholesterol accumulation increased with LDL density. Desialylated LDL of lower density was not atherogenic, and the densest sialylated LDL was capable of inducing a moderate increase in cellular cholesterol. However, this does not necessarily imply that the density or size of LDL particles are the primary determinants of their atherogenicity. As it was demonstrated previously, desialylated LDL is also characterized with decreased content of neutral sugars such as galactose and mannose and acetylated residues such as $\mathrm{N}$-acetylglucosamine..$^{[31]}$ It may be speculated that some extensively modified LDL particles may be deprived of not only sialic acid, but also of galactose residues, that usually become terminal saccharides in biantennary polysaccharide chains in apoB after desialylation. Such particles cannot be isolated from the total LDL preparation by $\mathrm{RCA}_{120}$ affinity chromatography and would contaminate non-bound LDL fractions that are generally thought to be non-modified, sialic acid-rich LDL. Assuming that diminished particle size, increased density, and loss of sugar residues are the processes characteristic for LDL modification and work in parallel, the densest part of non-bound (so-called sialylated) LDL would contain some amount of exceptionally modified LDL that could raise the intracellular cholesterol levels. Additionally, desialylated or sialylated LDL from diabetic patients did not differ significantly with respect to particle size from corresponding LDL fractions from healthy subjects; the difference, however, was dramatic with regard to the atherogenic effect. Therefore, the size or density of LDL particles may be regarded as the marker of LDL atherogenic modification, but seem to be secondary events reflecting the alterations in chemical composition of LDL that have occurred before. However, it may be speculated that smaller LDL particle size may lead to conformational changes in those apoB domains responsible for interaction with cell receptors. However, in this case, one should provide mechanistic explanations of the effect of LDL particle size on its binding, uptake, and internalization by cells; such explanation was provided only for desialylated LDL. ${ }^{[37]}$

Interestingly, LDL desialylation is assumed to be a systemic process, which affects not only LDL as a single target. Recently Koska et al. ${ }^{[38]}$ have reported that properly glycosylated apoC-III proteoform (i.e. apoprotein-bearing 2 sialic acid residues per every biantennary polysaccharide chain), in contrast to partially or totally desialylated proteoforms, is associated with beneficial lipid profile in prediabetic and type 2 diabetic patients. It is possible that apoCIII non-sialylated, monosialylated, and di-sialylated isoforms occur in circulation owing to desialylation (or another kind of deglycosylation), but not because of different pathways of posttranslational glycosylation in hepatocytes.

This study has certain limitations. First, it was of an experimental nature, and not aimed at estimating the clinical relevance of desialylated LDL with respect to diabetes and/or gender- and age-related differences, or association with metabolic control and therapy. Second, it was not possible to calculate the sample size prior to the study, because there were no available statistical data on variability of the studied parameters in healthy subjects and diabetic patients. Thus, only post hoc analysis was possible, which showed that the given sample size was sufficient to reach $96-100 \%$ statistical power at a $5 \%$ confidence level, with respect to observed inter-groups differences in LDL atherogenicity, electrophoretic mobility of desialylated LDL, and intragroup differences between sialylated and desialylated LDL particle sizes. Regardless, the results of our study conclude that there is a fraction of modified LDL along with native LDL in the blood of diabetic patients. This naturally occurring modified LDL is characterized by various alterations to its physicochemical properties. Our previous findings have shown that: (1) it is desialylated and non-enzymatically glycated lipoprotein; (2) it is characterized by lowered esterified cholesterol level and elevated content of lyso-phospholipids; and (3) it is atherogenic in terms of intracellular cholesterol accumulation. We believe our research presents new and significant abnormal peculiarities of this LDL: (1) it is a small, dense LDL; and (2) it is more electronegative than native LDL. Therefore, previously described atherogenic LDL fraction in diabetics can be regarded as multiple-modified LDL that may be assigned to have a significant role in early atherogenesis in diabetes mellitus. The origin and metabolic fate of this in vivo modified LDL remains to be studied.

\section{Acknowledgments}

We acknowledge the help of Dr. Olli Jaakkola and 
Dr. Tiina Solakivi (University of Tampere, Tampere, Finland) in methodologic supervising of preliminary studies with LDL density fractionation.

\section{Financial support and sponsorship}

This study was supported in part by Russian Ministry of Education and Science, Project\#RFMEFI61614X0010.

\section{Conflicts of interest}

There are no conflicts of interest.

\section{Patient consent}

All involved patients gave their consent forms.

\section{Ethics approval}

This study was approved by the local ethics committee of the Institute for Atherosclerosis Research, Skolkovo Innovation Center, Moscow, Russia.

\section{REFERENCES}

1. Ghosh P, Sahoo R, Vaidya A, Chorev M, Halperin JA. Role of complement and complement regulatory proteins in the complications of diabetes. Endocr Rev 2015;36:272-88.

2. Nathan DM, Bayless M, Cleary P, Genuth S, Gubitosi-Klug R, Lachin JM, Lorenzi G, Zinman B; DCCT/EDIC Research Group. Diabetes control and complications trial/epidemiology of diabetes interventions and complications study at 30 years: advances and contributions. Diabetes 2013;62:3976-86.

3. Moore DJ, Gregory JM, Kumah-Crystal YA, Simmons JH. Mitigating micro-and macro-vascular complications of diabetes beginning in adolescence. Vasc Health Risk Manag 2009;5:1015-31.

4. Aronson D, Edelman ER. Coronary artery disease and diabetes mellitus. Heart Fail Clin 2016;12:117-33.

5. Kones R. Primary prevention of coronary heart disease: integration of new data, evolving views, revised goals, and role of rosuvastatin in management. A comprehensive survey. Drug Des Devel Ther 2011;5:325-80.

6. Goldstein JL, Brown MS. A century of cholesterol and coronaries: from plaques to genes to statins. Cell 2015;161:161-72.

7. Shi H, Mao X, Zhong Y, Liu Y, Zhao X, Yu K, Zhu R, Wei Y, Zhu J, Sun H, Mao Y, Zeng Q. Lanatoside C promotes foam cell formation and atherosclerosis. Sci Rep 2016;6:20154.

8. Das A, Brown MS, Anderson DD, Goldstein JL, Radhakrishnan A. Three pools of plasma membrane cholesterol and their relation to cholesterol homeostasis. Elife 2014;3:02882.

9. Yang H, Mohamed AS, Zhou SH. Oxidized low density lipoprotein, stem cells, and atherosclerosis. Lipids Health Dis 2012;11:85.

10. Orekhov AN, Melnichenko AA, Sobenin IA. Approach to reduction of blood atherogenicity. Oxid Med Cell Longev 2014;2014:738679.

11. Bobryshev YV, Ivanova EA, Chistiakov DA, Nikiforov NG, Orekhov AN. Macrophages and their role in atherosclerosis: pathophysiology and transcriptome analysis. Biomed Res Int 2016;2016:9582430.

12. Zakiev ER, Sukhorukov VN, Melnichenko AA, Sobenin IA, Ivanova EA, Orekhov AN. Lipid composition of circulating multiple-modified low density lipoprotein. Lipids Health Dis 2016;15:134.

13. Sobenin, IA, Tertov VV, Koschinsky T, Bünting CE, Slavina ES, Dedov II, Orekhov AN. Modified low density lipoprotein from diabetic patients causes cholesterol accumulation in human intimal aortic cells. Atherosclerosis 1993;100:41-54.

14. Sobenin IA, Tertov VV, Orekhov AN. Characterization of chemical composition of native and modified low density lipoprotein occurring in the blood of diabetic patients. Int Angiol 1994;13:78-83.

15. Austin MA, Breslow JL, Hennekens CH, Buring JE, Willett WC, Krauss RM. Low-density lipoprotein subclass patterns and risk of myocardial infarction. JAMA 1988;260:1917-21.

16. Musliner TA, Krauss RM. Lipoprotein subspecies and risk of coronary disease. Clin Chem 1988;34:B78-83.

17. Packard CJ. Small dense low-density lipoprotein and its role as an independent predictor of cardiovascular disease. Curr Opin Lipidol 2006;17:412-7.

18. Ivanova EA, Bobryshev YV, Orekhov AN. LDL electronegativity index: a potential novel index for predicting cardiovascular disease. Vasc Health Risk Manag 2015;11:525-32.

19. Puavilai G, Chanprasertyotin S, Sriphrapradaeng A. Diagnostic criteria for diabetes mellitus and other categories of glucose intolerance: 1997 criteria by the Expert Committee on the Diagnosis and Classification of Diabetes Mellitus (ADA), 1998 WHO consultation criteria, and 1985 WHO criteria. World Health Organization. Diabetes Res Clin Pract 1999;44:21-6.

20. Lindgren FT. Preparative ultracentrifugal laboratory procedures and suggestions for lipoproten analysis. In: Perkins EG, editor. Analysis of lipids and lipoproteins. New York: American Oil Chemical Society; 1975. p. 205-24.

21. Tertov VV, Sobenin IA, Tonevitsky AG, Orekhov AN, Smirnov VN. Isolation of atherogenic modified (desialylated) low density lipoprotein from blood of atherosclerotic patients: separation from native lipoprotein by affinity chromatography. Biochem Biophys Res Commun 1990;167:1122-7.

22. Jaakkola O, Solakivi T, Ylä-Herttuala S, Nikkari T. Receptormediated binding and degradation of subfractions of human plasma low-density lipoprotein by cultured fibroblasts. Biochim Biophys Acta 1989;1005:118-22.

23. Jaakkola O, Solakivi T, Tertov VV, Orekhov AN, Miettinen TA, Nikkari T. Characteristics of low-density lipoprotein subfractions from patients with coronary artery disease. Coron Artery Dis 1993;4:379-85.

24. Hara A, Radin NS. Lipid extraction of tissues with a low-toxicity solvent. Anal Biochem 1978;90:420-6.

25. Siedel J, Hägele EO, Ziegenhorn J, Wahlefeld AW. Reagent for enzymatic determination of serum total cholesterol with improved lipolytic efficiency. Clin Chem 1983;29:1075-80.

26. Lowry OH, Rosebrough NJ, Farr AL, Randall BJ. Protein measurement with the Folin phenol reagent. $J$ Biol Chem 1951;193:265-75.

27. Lipid Research Clinics Program. In: Manual of Laboratory Operations, Lipid and Lipoprotein Analysis, DHEW Publication No. (NIH) 75628. 2nd ed. Washington, DC: U. S. Government Printing Office; 1974. p. 1-81.

28. Krauss RM, Burke DJ. Identification of multiple subclasses of plasma low density lipoproteins in normal humans. J Lipid Res 1982;23:97104.

29. Sobenin IA, Tertov VV, Orekhov AN. Optimization of the assay for sialic acid determination in low density lipoprotein. $J$ Lipid Res 1998;39:2293-9.

30. Tsai NW, Lee LH, Huang CR, Chang WN, Chang YT, Su YJ, Chiang YF, Wang HC, Cheng BC, Lin WC, Kung CT, Su CM, Lin YJ, Lu $\mathrm{CH}$. Statin therapy reduces oxidized low density lipoprotein level, a risk factor for stroke outcome. Crit Care 2014;18:R16.

31. Liu Y, Atkinson D. Enhancing the contrast of ApoB to locate the surface components in the 3D density map of human LDL. J Mol Biol 2011;405:274-83 
32. Aksenov DV, Medvedeva LA, Skalbe TA, Sobenin IA, Tertov VV, Gabbasov ZA, Popov EV, Orekhov AN. Deglycosylation of apo B-containing lipoproteins increase their ability to aggregate and to promote intracellular cholesterol accumulation in vitro. Arch Physiol Biochem 2008;114:349-56.

33. Nigam PK, Narain VS, Kumar A. Sialic acid in cardiovascular diseases. Indian J Clin Biochem 2006;21:54-61.

34. Zeljkovic A, Vekic J, Spasojevic-Kalimanovska V, Jelic-Ivanovic Z, Bogavac-Stanojevic N, Gulan B, Spasic S. LDL and HDL subclasses in acute ischemic stroke: prediction of risk and short-term mortality. Atherosclerosis 2010;210:548-54.

35. Oravec S, Krivosikova Z, Krivosik M, Gruber K, Gruber M, Dukát A, Gavorník P. Lipoprotein profile in patients who survive a stroke.
Neuro Endocrinol Lett 2011;32:496-501.

36. Albers JJ, Slee A, Fleg JL, O’Brien KD, Marcovina SM. Relationship of baseline HDL subclasses, small dense LDL and LDL triglyceride to cardiovascular events in the AIM-HIGH clinical trial. Atherosclerosis 2016;251:454-9.

37. Orekhov AN, Tertov VV, Sobenin IA, Smirnov VN, Via DP, Guevara J Jr, Gotto AM Jr, Morrisett JD. Sialic acid content of human low density lipoproteins affects their interaction with cell receptors and intracellular lipid accumulation. J Lipid Res 1992;33:805-17.

38. Koska J, Yassine H, Trenchevska O, Sinari S, Schwenke DC, Yen FT, Billheimer D, Nelson RW, Nedelkov D, Reaven PD. Disialylated apolipoprotein C-III proteoform is associated with improved lipids in prediabetes and type 2 diabetes. J Lipid Res 2016;57:894-905. 